\title{
A Bessel differential heat initial value problem in a reproducing kernel Hilbert space
}

framework

L. P. Castro and M. M. RodriguesS. Saitoh

Citation: 1637, (2014); doi: 10.1063/1.4904575

View online: http://dx.doi.org/10.1063/1.4904575

View Table of Contents: http://aip.scitation.org/toc/apc/1637/1

Published by the American Institute of Physics 


\title{
A Bessel differential heat initial value problem in a reproducing kernel Hilbert space framework
}

\author{
L.P. Castro*, M.M. Rodrigues* and S. Saitoh ${ }^{\dagger}$ \\ *CIDMA - Center for Research and Development in Mathematics and Applications, Department of \\ Mathematics, University of Aveiro, Campus Universitário de Santiago, 3810-193 Aveiro, Portugal \\ ${ }^{\dagger}$ Institute of Reproducing Kernels, Kawauchi-cho, 5-1648-16, Kiryu 376-0041, Japan
}

\begin{abstract}
For modified Bessel heat equations subjected to an initial condition, we investigate integral transforms with kernels related to the solutions of those equations by using the theory of reproducing kernels. In particular, a new framework within reproducing kernel Hilbert spaces is proposed where we construct the unique solutions of the corresponding initial value problems.
\end{abstract}

Keywords: Integral transform, isometric mapping, inversion formula, initial value problem, regularization, eigenfunction, eigenvalue, reproducing kernel, inverse problems

PACS: $02.30 . \mathrm{Gp}$; 02.30.Hq; 02.30.Jr; 02.30.Rz; 02.30.Sa; 02.30.Uu; 02.30.Zz

\section{INTRODUCTION}

Bessel functions are defined as particular solutions of linear differential equations of the second order. These are known as Bessel's equations and are often used in the modelling of real physical problems. In particular, linear PDE's describing various chemical transfer processes allow the existence of exact solutions expressed in terms of one special kind of Bessel's functions. Modelling of different process cases, from hydrodynamics, diffusion, heat transfer and other interdisciplinary topics, illustrate the wide applications of the Bessel's functions (see e.g. [9]).

In view of those interests, properties of integral transforms whose kernels are functions somehow associated with the fundamental solutions of heat equations of various types have been investigated extensively. For example, in [8], I. I. Hirschman and D. V. Widder developed an inversion and representation theory for transforms related to the classical heat equation; F. Cholewinski and D. T. Haimo, in [6], derived corresponding results for transforms arising from the generalized heat equation.

We will consider here the modified Bessel differential equations

$$
x^{2} \frac{d^{2} y}{d x^{2}}+x \frac{d y}{d x}-\left(x^{2}+v^{2}\right) y=0
$$

which have as solutions the modified Bessel functions of second kind, i.e., $y=K_{v}$. Consequently, $K_{v}$ are eigenfunctions of the corresponding second order differential, i.e.

$$
L_{x} K_{v}(x)=v^{2} K_{v}(x)
$$

with an eigenvalue $v^{2}$, where

$$
L_{x}=x^{2} \frac{d^{2}}{d x^{2}}+x \frac{d}{d x}-x^{2} .
$$

For pure imaginary values of $v$, the functions

$$
\exp \left(v^{2} t\right) K_{v}(x)
$$

are the solutions of the operator equation

$$
\left(\partial_{t}-L_{x}\right) u(t, x)=0 .
$$

For a pure imaginary $v=i \tau$, we have as eigenvalues associated to the second order differential (3), $v^{2}=-\tau^{2}$. We shall consider some general solutions of (5) by a suitable sum of the solutions (4). In order to consider a fully general sum, we shall consider the kernel form, for a nonnegative continuous function $\rho$,

$$
\mathscr{K}_{t}(x, y ; \rho)=\int_{0}^{+\infty} \exp \left\{-\tau^{2} t\right\} K_{i \tau}(x) K_{i \tau}(y) \rho(\tau) d \tau .
$$


Note that we are considering the integral with absolutely convergence for the kernel form. In addition, note that $K_{i \tau}(x)$ is a real valued function and the kernel $\mathscr{K}_{t}(x, y ; \rho)$ is symmetric.

The full general solutions of the equation (5) may be represented in the integral form

$$
u(t, x)=\int_{0}^{+\infty} \exp \left\{-\tau^{2} t\right\} K_{i \tau}(x) F(\tau) \rho(\tau) d \tau
$$

for the functions $F$ satisfying

$$
\int_{0}^{+\infty} \exp \left\{-\tau^{2} t\right\}|F(\tau)|^{2} \rho(\tau) d \tau<\infty
$$

Then, the solution $u(t, x)$ of (5) satisfying the initial condition

$$
u(0, x)=F(x)
$$

will be obtained by taking $t \rightarrow 0$ in (7). However, this procedure is not trivial and we will propose here a convenient structure so that this will be possible. Here, (6) is a reproducing kernel and so, in order to analyze the logic above, we will therefore need the theory of reproducing kernels.

\section{SOLVABILITY OF THE INITIAL VALUE PROBLEM IN A REPRODUCING KERNEL FRAMEWORK}

We start by recalling that the modified Bessel function of second kind $K_{i \tau}$ is an eigenfunction of a second order differential. Namely,

$$
L_{x} K_{i \tau}(x)=-\tau^{2} K_{i \tau}(x), \quad x>0,
$$

where $L_{x}$ is given in (3).

Then, we form the reproducing kernel

$$
\mathscr{K}(x, y ; \rho)=\int_{0}^{+\infty} K_{i \tau}(x) K_{i \tau}(y) \rho(\tau) d \tau
$$

and consider the reproducing kernel Hilbert space $H_{\mathscr{K}(\rho)}\left(\mathbb{R}^{+}\right)$admitting the kernel $\mathscr{K}(x, y ; \rho)$. In particular, note that

$$
\mathscr{K}_{t}(x, y ; \rho) \in H_{\mathscr{K}(\rho)}\left(\mathbb{R}^{+}\right), \quad y>0 .
$$

Theorem 0.1 For any $f \in H_{\mathscr{K}(\rho)}\left(\mathbb{R}^{+}\right)$, the solution $u_{f}(t, x)$ of the initial value problem

$$
\left\{\begin{array}{l}
\left(\partial_{t}-x^{2} \partial_{x}^{2}-x \partial_{x}+x^{2}\right) u_{f}(t, x)=0, \quad t>0 \\
u_{f}(0, x)=f(x)
\end{array}\right.
$$

exists uniquely and is given by

$$
u_{f}(t, x)=\left(f(\cdot), \mathscr{K}_{t}(\cdot, x ; \rho)\right)_{H_{\mathscr{K}(\rho)}\left(\mathbb{R}^{+}\right)} .
$$

As a preliminar observation, we stress that the initial value presented in (12) should be interpreted as

$$
\begin{aligned}
\lim _{t \rightarrow+0} u_{f}(t, x) & =\lim _{t \rightarrow+0}\left(f(\cdot), \mathscr{K}_{t}(\cdot, x ; \rho)\right)_{H_{\mathscr{K}}(\rho)}\left(\mathbb{R}^{+}\right) \\
& =(f(\cdot), \mathscr{K}(\cdot, x ; \rho))_{H_{\mathscr{K}}(\rho)}\left(\mathbb{R}^{+}\right) \\
& =f(x)
\end{aligned}
$$

whose existence is ensured and the limit is in the uniform convergence sense on any subset of $\mathbb{R}^{+}$such that $\mathscr{K}(x, x ; \rho)$ is bounded. Also, in the last theorem, the completeness property of the solutions $u_{f}(t, x)$ of (12) satisfying the initial value $f$ may be derived by the reproducing kernel Hilbert space admitting the kernel

$$
k(x, t ; y, \tau ; \rho):=\left(\mathscr{K}_{\tau}(\cdot, y ; \rho), \mathscr{K}_{t}(\cdot, x ; \rho)\right)_{H_{\mathscr{K}(\rho)}\left(\mathbb{R}^{+}\right)} .
$$


Moreover, in our method, we see that the existence problem of the initial value problem is based on the eigenfunctions and we are constructing the desired solution satisfying the initial condition in consideration. Furthermore, by considering the linear mapping of (13) with various situations, we will be able to obtain various inverse problems looking for initial values $f$ from different data output of $u_{f}(t, x)$.

Proof: We start by realizing that the kernel $\mathscr{K}_{t}(x, y ; \rho)$ satisfies the operator equation in (12) for any fixed $y$. This is the case because the functions

$$
\exp \left\{-\tau^{2} t\right\} K_{i \tau}(x)
$$

satisfy the operator equation and it is the summation. Similarly, the function $u_{f}(t, x)$ defined by (13) is the solution of the operator equation in (12). Note that the kernels $\mathscr{K}_{t}(x, y ; \rho)$ and $\mathscr{K}(x, y ; \rho)$ are symmetric functions.

In order to verify the initial value condition, note first the important general property

$$
\mathscr{K}_{t}(y, x ; \rho) \ll \mathscr{K}(y, x ; \rho) .
$$

I.e., $\mathscr{K}(y, x ; \rho)-\mathscr{K}_{t}(y, x ; \rho)$ is a positive definite quadratic form function and we have

$$
H_{\mathscr{K}_{t}(\rho)} \subset H_{\mathscr{K}(\rho)}\left(\mathbb{R}^{+}\right)
$$

and

$$
\|f\|_{H_{\mathscr{K}(\rho)}\left(\mathbb{R}^{+}\right)}=\lim _{t \rightarrow+0}\|f\|_{H_{\mathscr{K}}(\rho)},
$$

for any function $f \in H_{\mathscr{K}_{t}(\rho)}$ (in the sense of non-decreasing norm convergence [2]). Thus, in order to verify the initial value identity (or the crucial point in (14)), we have just to analyse that

$$
\begin{aligned}
\left\|\mathscr{K}(y, x ; \rho)-\mathscr{K}_{t}(y, x ; \rho)\right\|_{H_{\mathscr{K}(\rho)}\left(\mathbb{R}^{+}\right)}^{2} & =\mathscr{K}(x, x ; \rho)-2 \mathscr{K}_{t}(x, x ; \rho)+\left\|\mathscr{K}_{t}(y, x ; \rho)\right\|_{H_{\mathscr{K}(\rho)}\left(\mathbb{R}^{+}\right)}^{2} \\
& \leq \mathscr{K}(x, x ; \rho)-2 \mathscr{K}_{t}(x, x ; \rho)+\left\|\mathscr{K}_{t}(y, x ; \rho)\right\|_{H_{\mathscr{K}_{t}(\rho)}} \\
& =\mathscr{K}(x, x ; \rho)-\mathscr{K}_{t}(x, x ; \rho),
\end{aligned}
$$

and identify that this converges obviously to zero as $t \rightarrow+0$.

The uniqueness property of the initial value problem is depending on the completeness of the family of functions

$$
\left\{\mathscr{K}_{t}(\cdot, x ; \rho): x \in \mathbb{R}^{+}\right\}
$$

in $H_{\mathscr{K}(\rho)}\left(\mathbb{R}^{+}\right)$. Moreover, this completeness property is derived from Theorem 0.3 below.

\section{IDENTIFICATION OF THE REPRODUCING KERNEL HILBERT SPACES}

In the theorem of the last section, the realization of the reproducing kernel Hilbert space $H_{\mathscr{K}(\rho)}\left(\mathbb{R}^{+}\right)$is a crucial point. For this purpose, we are interested in the calculation of the kernel $\mathscr{K}(x, y ; \rho)$. Here, we would like to refer that such concrete integral formulas have a great tradition in some research schools as we can see in the interesting books $[10,11,12,13]$.

From [7], we have for our case:

$$
\int_{0}^{+\infty} K_{i \tau}(x) K_{i \tau}(y) \tau \tanh (\pi \tau) d \tau=\frac{\pi}{2}(x y)^{1 / 2}(x+y)^{-1} \exp (-x-y),
$$

$y>0,|\arg (x)|<\pi$ (see formula 12.1.4, in [7], p. 175). Moreover,

$$
\int_{0}^{+\infty} K_{i \tau}(x) K_{i \tau}(y) \cosh (\alpha \tau) d \tau=\frac{1}{2} \pi K_{0}\left(\sqrt{x^{2}+y^{2}+2 x y \cos (\alpha)}\right), \quad y>0,
$$

with $|\operatorname{Re}(\alpha)|+|\arg (x)|<\pi$ (see [7], formula 12.1.7, p. 175).

Recall that

$$
K_{v}(z)=\int_{0}^{+\infty} \exp (-z \cosh (w)) \cosh (v w) d w
$$


with $|\arg (z)|<\frac{\pi}{2}$.

Then,

$\int_{0}^{+\infty} K_{i \tau}(x) K_{i \tau}(y) \tau \sinh (\pi \tau) \Gamma(\lambda+i \tau) \Gamma(\lambda-i \tau) d \tau=2^{\lambda-1} \pi^{3 / 2}(x y)^{\lambda}(y+x)^{-\lambda} \Gamma\left(\lambda+\frac{1}{2}\right) K_{\lambda}(y+x), y>0$,

$|\arg (x)|<\pi, \operatorname{Re}(\lambda)>0$ (see formula 12.1.12, p. 176, in [7]), and

$$
\int_{0}^{+\infty} K_{i \tau}(x) K_{i \tau}(y) \tau\left(\tau^{2}+n^{2}\right)^{-1} \sinh (\pi \tau) d \tau=\frac{\pi^{2}}{2} I_{n}(x) K_{n}(y), \quad y>x>0, \quad n=0,1,2,3, \ldots,
$$

where $I_{n}$ is the modified Bessel function of the first kind (cf. [7], formula 12.1.8, p. 176).

Moreover,

$$
\int_{0}^{+\infty} K_{i \tau}(x) K_{i \tau}(y) \tau \sinh (2 \pi \tau) \Gamma(\lambda+i \tau) \Gamma(\lambda-i \tau) d \tau=\frac{2^{\lambda} \pi^{5 / 2}}{\Gamma\left(\frac{1}{2}-\lambda\right)}\left(\frac{x y}{|y-x|}\right)^{\lambda} K_{\lambda}(|y-x|),
$$

$y>0, x>0,0<\operatorname{Re}(\lambda)<\frac{1}{2}$ (see [7], formula 12.1.13, p. 176),

$$
\int_{0}^{+\infty} K_{i \tau}(x) K_{i \tau}(y) \tau \sinh (\pi \tau) \Gamma\left(\lambda+i \frac{\tau}{2}\right) \Gamma\left(\lambda-i \frac{\tau}{2}\right) d \tau=2 \pi^{2}\left(\frac{x y}{2 \sqrt{y^{2}+x^{2}}}\right)^{2 \lambda} K_{2 \lambda}\left(\sqrt{y^{2}+x^{2}}\right),
$$

$y>0,|\arg (x)|<\frac{\pi}{2}, \operatorname{Re}(\lambda)>0$ (see formula 12.1.14, p. 177), and

$$
\int_{0}^{+\infty} \frac{K_{i \tau}(x) K_{i \tau}(y) \tau \tanh (\pi \tau)}{\Gamma\left(\frac{3}{4}+i \frac{\tau}{2}\right) \Gamma\left(\frac{3}{4}-i \frac{\tau}{2}\right)} d \tau=\frac{1}{2}\left(\frac{\pi x y}{y^{2}+x^{2}}\right)^{1 / 2} \exp \left(-\sqrt{y^{2}+x^{2}}\right),
$$

$y>0,|\arg (x)|<\frac{\pi}{2}$ (see formula 12.1.15, p. 177).

In addition,

$$
\int_{0}^{+\infty} K_{i \tau}(x) K_{i \tau}(y) K_{i \tau}(z) \tau \sinh (\pi \tau) d \tau=\frac{\pi^{2}}{4} \exp \left[-\frac{z}{2}\left(\frac{x}{y}+\frac{y}{x}+\frac{x y}{z^{2}}\right)\right],
$$

$|\arg (x)|+|\arg (y)|<\frac{\pi}{2}, z>0$ (see [7], formula 12.1.9, p. 176), and

$$
\int_{0}^{+\infty} K_{(i \tau) / 2}(x) K_{(i \tau) / 2}(y) K_{i \tau}(z) \tau \sin ((\pi \tau) / 2) d \tau=\frac{\pi^{2} z}{2 \sqrt{z^{2}+4 x y}} \exp \left[-\frac{(x+y) \sqrt{z^{2}+4 x y}}{2 \sqrt{x y}}\right]
$$

$|\arg (x)|+|\arg (y)|<\pi, z>0$ (cf. [7], formula 12.1.10, p. 176).

We would like to remark that the index integral transformation by using Bessel functions as kernels was introduced and studied by Titchmarsh (see [17]). Moreover, in [18], Wimp introduced an index transform over parameters of the Meijer G-functions.

We have just seen many concrete forms of the reproducing kernels (and, among these, we identify very complicated structures of the related reproducing kernel Hilbert spaces). For the point of view of the theory of reproducing kernels, their realizations will give interesting research topics which will have interest by their own. Here, we shall now discuss a somehow prototype example which is originated by the first situation above.

From the identity (18), we see that the images $f(z)$ of the integral transform

$$
f(z)=\int_{0}^{\infty} F(\tau) K_{i \tau}(z) \tau \tanh (\pi \tau) d \tau, \quad z=x+i y
$$

for the $L_{2}(0, \infty)$ functions $F(\tau)$ satisfying

$$
\int_{0}^{\infty}|F(\tau)|^{2} \tau \tanh (\pi \tau) d \tau<\infty
$$


are representable in the form

$$
f(z)=\frac{\pi}{2} z^{1 / 2} e^{-z} f_{1}(z)
$$

for some Szegö function space member $f_{1}(z)$ on the right hand complex plane satisfying

$$
\int_{-\infty}^{\infty}\left|f_{1}(i y)\right|^{2} d y<\infty
$$

Here, $f_{1}(i y)$ means the Fatou's non-tangential boundary values of the Szegö space of analytic functions on the right hand half complex plane. This result may be derived from the complex reproducing kernel that is derived from (18),

$$
K(z, \bar{u})=\frac{\pi}{2} z^{1 / 2} e^{-z} \overline{u^{1 / 2} e^{-u}} \frac{1}{z+\bar{u}}, \quad z=x+i y,
$$

on the right half complex plane. So, in particular, there is no problem for the multiple values of the fractional power on the right half complex plane. Thus, we obtain the isometric identity

$$
\|f\|_{H_{K}}^{2}=\frac{\pi}{2}\left\|f_{1}\right\|_{H_{S}}^{2}=\frac{1}{4} \int_{-\infty}^{+\infty}|f(i y)|^{2} \frac{d y}{y} .
$$

For full details on this point, we would like to refer the reader to [15].

Thus, we were able to realize the important reproducing kernel Hilbert space concretely and analytically. Meanwhile, we recall that we are also interested in the kernel forms $\mathscr{K}_{t}$ and $k$. These calculations will create new integral formulas.

We have to analyze and realize the corresponding reproducing kernel Hilbert spaces. However, we can apply a quite general formula by the Aveiro discretization method in $[3,4]$ (where numerical experiments are also given):

Proposition 0.2 For a uniqueness set $\left\{x_{j}\right\}$ for the reproducing kernel Hilbert space $H_{K}$ of a general set E satisfying the linearly independence of $K\left(\cdot, x_{j}\right)$ for any finite number of the points $x_{j}$, it holds

$$
\|f\|_{H_{K}}^{2}=\lim _{n \rightarrow \infty} \sum_{j=1}^{n} \sum_{j^{\prime}=1}^{n} f\left(x_{j}\right) \widetilde{a_{j j^{\prime}}} \overline{f\left(x_{j^{\prime}}\right)}
$$

with $\widetilde{a_{j j^{\prime}}}$ being the element of the complex conjugate inverse of the positive definite Hermitian matrix formed by $a_{j j^{\prime}}=K\left(x_{j}, x_{j^{\prime}}\right)$.

In Proposition 0.2, for the uniqueness set of the space, if the reproducing kernel is analytical, then, the criteria will be very simple by the identity theorem of analytic functions, as in the present case. For the Sobolev space case, we have to consider some dense subset of $E$ for the uniqueness set. Meanwhile, the linearly independence will be easily derived from the integral representations of the kernels.

For applying Proposition 0.2, we need only to consider the following result.

Theorem 0.3 In the integral transform induced from (11),

$$
\left\{K_{i \tau}(x) ; x \in \mathbb{R}^{+}\right\}
$$

is complete in $L^{2}\left(\mathbb{R}^{+}, \rho(\tau) d \tau\right)$. For any different points $\left\{x_{j}\right\}_{j=1}^{n}\left(x_{j}>0\right)$,

$$
\left\{K_{i \tau}\left(x_{j}\right)\right\}_{j=1}^{n}
$$

are linearly independent.

Proof: The completeness is directly arising from the integral representation of $K_{v}(z)$ and the linear independence is originated from the asymptotic expansion

$$
K_{\alpha}(x) \sim \sqrt{\frac{\pi}{2 x}} e^{-x}\left[1+\sum_{n=1}^{\infty} \frac{(\alpha, n)}{(2 x)^{n}}\right], \quad|\arg x|<\frac{3}{2} \pi,
$$


where

$$
(\alpha, n)=(-1)^{n}\left(\alpha+\frac{1}{2}\right)_{n}\left(-\alpha+\frac{1}{2}\right)_{n} n !
$$

(cf. [1], formula (4.12.6), p. 223).

As a final remark, about general initial value problems, we would like to point out that in [5] we have proposed a general machinery based on reproducing kernel methods for dealing with the existence and construction of solutions of classes of initial value problems for somehow general operators in abstract function spaces. Anyway, that is an abstract method which -in a sense- assumes the validity of certain important steps which need to be validated when considering specific cases.

\section{ACKNOWLEDGMENTS}

This work was supported by Portuguese funds through the CIDMA - Center for Research and Development in Mathematics and Applications, and the Portuguese Foundation for Science and Technology ("FCT-Fundação para a Ciência e a Tecnologia”), within project PEst-OE/MAT/UI4106/2014.

\section{REFERENCES}

1. G.E. Andrews, R. Askey and R. Roy, Special Functions, Encyclopedia of Mathematics and its Applications, Vol.71, Cambridge University Press, Cambridge, 2009.

2. N. Aronszajn, Theory of reproducing kernels, Trans. Amer. Math. Soc. 68, 337-404 (1950).

3. L.P. Castro, H. Fujiwara, M.M. Rodrigues, S. Saitoh and V.K. Tuan, Aveiro Discretization Method in Mathematics: A New Discretization Principle, in Mathematics without Boundaries: Surveys in Pure Mathematics, edited by P. Pardalos and M. Themistocles, Springer-Verlag, New York, 52 pp., in press, ISBN 978-1-4939-1105-9.

4. L.P. Castro, H. Fujiwara, T. Qian and S.Saitoh, How to catch smoothing properties and analyticity of functions by computers? in Mathematics without Boundaries: Surveys in Interdisciplinary Mathematics, edited by P. Pardalos and T.M. Rassias, Springer-Verlag, New York, 15 pp., in press, ISBN 978-1-4939-1123-3.

5. L.P. Castro, M.M. Rodrigues and S. Saitoh, A fundamental theorem on initial value problems by using the theory of reproducing kernels, Complex Anal. Oper. Theory, 12pp., to appear, DOI:10.1007/s11785-014-0375-1.

6. F. Cholewinski and D.T. Haimo, Integral representations of solutions of the generalized heat equation, Ill. J. Math. 10, 623-638 (1966).

7. A. Erdélyi, W. Magnus, F. Oberhettinger and F. Tricomi, Tables of Integral Transforms. Vol I, II, Bateman Manuscript Project, California Institute of Technology, McGraw-Hill Book Company, New York-Toronto-London, 1954.

8. I. Hirschman and D. Widder, The Convolution Transform, Princeton Mathematical Series, No. 20, Princeton University Press, Princeton, 1955.

9. T. Petrova, Application of Bessel's functions in the modelling of chemical engineering processes, Bulgarian Chemical Communications 41(4), 343-354 (2009).

10. A.P. Prudnikov, Yu.A. Brychkov and O.I. Marichev, Integrals and Series, Vol. 3: More Special Functions, Gordon and Breach Publisher, New York, 1990.

11. A.D. Polyanin, Handbook of Linear Partial Differential Equations for Engineers and Scientists, Chapman \& Hall/CRC Press, Boca Raton, FL, 2002

12. A.D. Polyanin and V.F. Zaitsev, Handbook of Exact Solutions for Ordinary Differential Equations, CRC Press, Boca Raton, FL, 2003.

13. A.D. Polyanin and A.V. Manzhirov, Handbook of Integral Equations, CRC Press, Boca Raton, FL, 2008.

14. S. Saitoh, Hilbert spaces induced by Hilbert space valued functions, Proc. Amer. Math. Soc. 89, 74-78 (1983).

15. S. Saitoh, Integral Transforms, Reproducing Kernels and their Applications, Pitman Research Notes in Mathematics Series 369, Addison Wesley Longman, Harlow, 1997.

16. S. Saitoh, Theory of reproducing kernels: Applications to approximate solutions of bounded linear operator functions on Hilbert spaces, Amer. Math. Soc. Transl., Ser.2 230, 107-134 (2010).

17. E.C. Titchmarsh, Eigenfunction Expansions Associated with Second-Order Differential Equations, Part I, Clarendon Press, Oxford, 1946.

18. J. Wimp, A class of integral transforms, Proc. Edinburgh Math Soc., II Ser. 14(1), 33-44 (1964). 